\title{
DSR-GUP Black Hole based on COW experiment and Einstein-Bohr's photon box
}

\author{
Nasrin Farahani ${ }^{1}$, Hassan Hassanabadi ${ }^{1,2, a}$, Jan Kř́ž ${ }^{2}$, Won Sang Chung ${ }^{3}$, Saber Zarrinkamar ${ }^{4}$ \\ ${ }^{1}$ Faculty of Physics, Shahrood University of Technology, Shahrood, Iran \\ 2 Department of Physics, University of Hradec Králové, Rokitanského 62, 50003 Hradec Králové, Czechia \\ ${ }^{3}$ Department of Physics and Research Institute of Natural Science, College of Natural Science, Gyeongsang National University, Jinju 660-701, \\ Korea \\ ${ }^{4}$ Department of Basic Sciences, Garmsar Branch, Islamic Azad University, Garmsar, Iran
}

Received: 7 March 2020 / Accepted: 21 July 2020 / Published online: 3 August 2020

(C) The Author(s) 2020

\begin{abstract}
In this paper, by studying the COW experiment and the Einstein Bohr's photon box, we investigate the associated modified phase shift and Hawking temperature. Next, we comment on the effective Newton constant suggested by the doubly special relativity based on the generalized uncertainty principle.
\end{abstract}

\section{Introduction}

Recently, it has been proposed that the quantum gravity effect might modify the ordinary Heisenberg uncertainty principle (HUP). It means that HUP in the quantum gravity changes to the generalized uncertainty principle (GUP) [1,2]. The GUP can be derived from String theory [3], Gedanken experiment, and non-commutative geometry $[4,5]$. Also, we know that GUP has a minimal uncertainty in the position at Planck length order [6]. There are many articles discussing the modified Unruh temperature in the presence of GUP and its Heisenberg algebra in the (Anti)-Snyder models [7]. Some models introduce doubly special relativity based on generalized uncertainty principle (DSR-GUP). In addition to, the Planck dimensions are partial the invariancy [8]. Various generalizations of HUP are introduced in DSR $[9,10]$. The experiment by Colella, Overhauser, and Werner (COW) [11], shows how gravity appears in the realm of quantum theory [12]. This phenomenon which is studied in a series of experiments could show the quantum interference caused by gravity incorporates the gravitational interference pattern of thermal neutrons. When two beams in a gravitational field come together in different path, they have a different phase called the phase shift. We intend to obtain the modified phase shift based on DSR-GUP [13]. As argued in the following,

a e-mail: hha1349@gmail.com (corresponding author) we can derive the modified Hawking temperature [14] for the DSR-GUP based on Einstein-Bohr's photon box [15, 16]. In the second section, by introducing DSR-GUP, we would calculate the wave-particle duality based on modified HUP. Furthermore, we obtain the modified phase shift for DSRGUP based on the COW experiment. In the third section, we calculate the modified Hawking temperature, mass, specific heat, and entropy based on Einstein Bohr's photon box for DSR-GUP black hole. In conclusions, we discuss and compare the mass and thermodynamics properties at different temperatures.

\section{COW experiment based on DSR-GUP}

\subsection{Modified wave-particle duality based on DSR-GUP}

In the Planck dimension, the HUP is modified to GUP which includes a new parameter $\beta$ of order Planck length [17].The generalized uncertainty principle, corresponds to generalized commutation relations [18].

$$
[\hat{X}, \hat{P}]=\mathrm{i} \hbar\left(1+\beta^{2} \hat{P}^{2}\right) \text {. }
$$

In DSR-GUP formalism, Planck dimensions are invariant $[8,19]$.

$$
[\hat{X}, \hat{P}]=\mathrm{i} \hbar\left(1-\frac{|\hat{P}|}{\kappa}\right)^{2},
$$

where $\kappa$ denotes the Planck momentum [8].

$$
\Delta X \Delta P \geq \frac{\hbar}{2}\left(1-2 \frac{\langle\hat{P} \mid\rangle}{\kappa}+\frac{(\Delta P)^{2}}{\kappa^{2}}\right) .
$$


The representations $\hat{X}$ and $\hat{P}$ in Eq. (2) are defined as

$\hat{X}=\hat{x}, \quad \hat{P}=\frac{\hat{p}}{1+\frac{|\hat{p}|}{\kappa}}$.

Also, the representation $\hat{p}$ based on $\hat{P}$ is defined as following with $\frac{|P|}{\kappa} \leq 1$

$\hat{p}=\frac{\hat{P}}{1-\frac{|\hat{P}|}{\kappa}}$.

Which will be considered in this paper and the commutation relations are not necessarily the same as Eq. (2). Furthermore, we assume a general commutator as [20]

$[\hat{X}, \hat{P}]=\mathrm{i} \hbar \vartheta$,

where $\vartheta=\vartheta(\hat{P})$ is a function of momentum. The average value of $\vartheta$, should have a lower bound as

$\Delta X \geq \frac{\vartheta}{\Delta P} \geq \frac{1}{\kappa}$.

Considering the relation between the wave-particle duality and HUP, let us obtain the modified de-Broglie formula from the DSR-GUP commutator Eq. (6) [21,22].

The modified de-Broglie formula based on DSR-GUP is given by $[20,23,24]$

$\lambda=\frac{2 \pi \hbar}{\left(\frac{\hat{P}}{1-\frac{\hat{P} \mid}{\kappa}}\right)}$.

Considering the previous equation, we obtain

$$
\begin{aligned}
\frac{\mathrm{d}}{\mathrm{d} \hat{P}}\left(\frac{2 \pi}{\lambda}\right) & =\hbar^{-1} \frac{\mathrm{d}}{\mathrm{d} \hat{P}}\left(\frac{\hat{P}}{1-\frac{|\hat{P}|}{\kappa}}\right) \\
& =\hbar^{-1} \frac{1}{\left(1-\frac{|\hat{P}|}{\kappa}\right)^{2}}=\hbar^{-1} \vartheta^{-1} .
\end{aligned}
$$

As we know $\hat{k}=k(\hat{P})$. We now introduce a commutation relation of the form

$[\hat{x}, \hat{k}]=\mathrm{i}, \quad[\hat{X}, \hat{P}]=[\hat{x}, \hat{k}] \frac{\mathrm{d} \hat{P}}{\mathrm{~d} \hat{k}}=\mathrm{i} \frac{\mathrm{d} \hat{P}}{\mathrm{~d} \hat{k}}$.

Comparing Eq. (6) with (10), we have [20]

$\frac{\mathrm{d} \hat{k}}{\mathrm{~d} \hat{P}}=\hbar^{-1} \vartheta^{-1}$,

or

$k(\hat{P})=\hbar^{-1} \int \vartheta^{-1} \mathrm{~d} \hat{P}$.

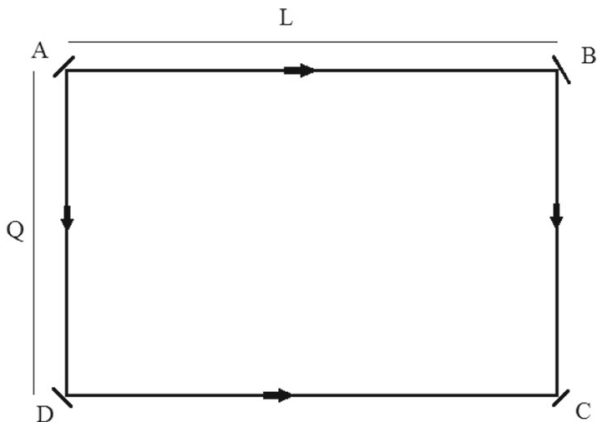

Fig. 1 COW experiment

in the next section, we use this formula to find a modified phase shift based on DSR-GUP.

\subsection{Modified phase shift in DSR-GUP formalism}

The experiment of Colella, Overhauser, and Werner (COW experiments for short), $\mathrm{COW}$ experiments on neutron interference in a gravitational field, was the first experiment in which both gravitational and quantum effects where incorporated [25]. In 1975 the first observation of gravitationallyinduced quantum interference was made in a neutron interferometer experiment [26]. This experiment was based on the phase shift induced by a gravitational field in the field of quantum interference of two neutron beams in different directions. As shown in Fig. 1, two neutron beams in two paths $\mathrm{ABC}$ and $\mathrm{ADC}$ influenced by gravitational field by considering DSR-GUP have a phase shift that depends on DSR-GUP [13]. Therefore, we investigate the quantum mechanical theory describing the COW experiment with the assumption "DSR-GUP" background described by Eq. (2). We intend to investigate the effects of DSR-GUP formulation of the COW experiment [27]. The phase shift formula previously reported in experiments is [20]

$\Delta \varphi=\frac{m g A}{\hbar v}$.

Now, by considering the phase shift of Ref. [20], we have

$\Delta \varphi^{\prime}=2 \pi\left(\frac{L}{\lambda_{2}}-\frac{L}{\lambda_{1}}\right)=L \Delta k=L \frac{\Delta k}{\Delta P} \Delta P$,

where $\Delta P$ is the difference in momentum between two horizontal beams in Fig. 1. Since $\Delta P$ is a small quantity, by considering Eqs. (11) and (13), we have

$\Delta \varphi^{\prime} \approx L \frac{d k}{d P} \Delta P=\hbar^{-1} \vartheta^{-1} L \Delta P$.

The neutron beams obey the energy conservation law and by assumption $v=\left(P_{1}+P_{2}\right) / 2 m$, we obtain

$m g Q=\frac{P_{2}^{2}-P_{1}^{2}}{2 m}=v \Delta P$. 
By inserting Eq. (15) into (14), we have

$\Delta \varphi^{\prime}=\frac{m g Q L}{\vartheta \hbar v}=\frac{m g Q L}{\left(1-\frac{|P|}{\kappa}\right)^{2} \hbar v}=\frac{m g^{\prime} A}{\hbar v}$.

We obtained the modified phase shift from DSR-GUP, where $g^{\prime}=g / \vartheta, A=Q L$. When $\vartheta=1$, Eq. (16) reduces to the ordinary form of Eq. (12).

In the special case, we suppose that the deformed parameter, for the $i$ th case is $\kappa_{i}$ and the modified phase shift is $\Delta \varphi_{i}$, and for the $j$ th case, we have $\kappa_{j}$ and $\Delta \varphi_{j}$, therefore, we have

$\Delta \varphi_{j}-\Delta \varphi_{i}=\frac{m g_{j}^{\prime} A}{\hbar v}-\frac{m g_{i}^{\prime} A}{\hbar v}$,

or equally

$\delta \varphi=\frac{m A \delta g^{\prime}}{\hbar v}$,

where $\delta \varphi=\Delta \varphi_{j}-\Delta \varphi_{i}$ and $\delta g^{\prime}=g_{j}^{\prime}-g_{i}^{\prime}$. Thus, the intensity in the interference region as a function of $g^{\prime}$ represents a periodic behavior. We call $\delta g^{\prime}$ the difference in $g^{\prime}$ needed to produce successive maxima.

We can easily show that

$\frac{m A \delta g^{\prime}}{\hbar v}=\pi \Longrightarrow \delta g^{\prime}=\frac{\hbar \nu \pi}{m A}$ or $\frac{m A}{\hbar \nu \pi}=\frac{v_{j}-v_{i}}{g}$

Then, we obtain

$\frac{m g A}{\hbar \nu \pi}=\left(\frac{1}{\left(1-\frac{|P|}{\kappa_{j}}\right)^{2}}-\frac{1}{\left(1-\frac{|P|}{\kappa_{i}}\right)^{2}}\right)$

where $\kappa_{i}$ and $\kappa_{j}$ are the deformed parameters.

\section{Modified Hawking temperature based on DSR-GUP}

Einstein suggested assuming a box with perfectly reflecting walls containing photons. Inside of the box, a clock mechanism could open and close a shutter at a predetermined moment $t$ for a time interval $\Delta t$ short enough to let just one photon irradiate [20]. Therefore, when $\Delta t$ tends to zero, the photon emission time can be exactly known. Before and after emission, we are to weigh the box and from the difference in the weight we could accurately determine the energy $E$ of the photon. Thus, as Einstein mentioned, the photon emitted violated the Heisenberg relation (HUP) [28,29].

On the other hand, Bohr believed that the problem originates from the nature of time since time is not a quantum mechanical observable and we could not use it from HUP.
We know that the gravitational force $(g \Delta m)$ related to the mass, acting a time $t$ yields the momentum change

$\Delta P=g \Delta m t$

By substituting $\triangle P$ into HUP we have

$\Delta X \geq \frac{\hbar}{\Delta m g t}$.

We know that for the clock in the box the period time is given by [20]

$\Delta t=\frac{g \Delta X}{c^{2}} t$

By inserting Eqs. (23) into (22) we have

$\Delta t \geq \frac{\hbar}{\Delta m c^{2}}$.

Using Eq. (24) and replacing into $\Delta E \Delta t \geq \hbar$ we obtain

$\Delta E \geq \frac{\hbar c^{2}}{g t \Delta X}$

Now, we simply find

$$
\begin{aligned}
& \frac{\Delta P_{\min }}{t}=\frac{\vartheta \hbar}{t \Delta X} \leq g \Delta m, \quad \Delta P_{\min }=\frac{\vartheta \hbar}{\Delta X} . \\
& \vartheta \hbar=\Delta X \Delta P_{\min } \leq \Delta m(g \Delta X) t,
\end{aligned}
$$

By replacing Eq. (23) into the previous equation we have

$\vartheta \hbar \leq c^{2} \Delta m \Delta t=\Delta E \Delta t$

Then, we have

$\Delta E^{D S R-G U P} \geq \frac{\vartheta \hbar c^{2}}{g \Delta X t}=\frac{\hbar c^{2}}{g^{\prime} \Delta X t}$,

where $g^{\prime}=g / \vartheta$. The time uncertainty becomes

$\Delta t^{\prime}=\frac{g^{\prime} \Delta X}{c^{2}} t, \quad \Delta t^{\prime}=\Delta t / \vartheta$

where $G^{\prime}=G / \vartheta$ is just the one proposed in Refs. [20,27]. Meanwhile, we obtain the modified Schwarzschild metric and modified Newton constant inspired by DSR-GUP as

$$
\begin{aligned}
d s^{2}= & -\left(1-\frac{2 G^{\prime} M}{c^{2} r}\right) c^{2} d t^{2}+\left(1-\frac{2 G^{\prime} M}{c^{2} r}\right)^{-1} d r^{2} \\
& +r^{2} d \theta^{2}+r^{2} \sin ^{2} \theta d \phi^{2}
\end{aligned}
$$

Now we consider a DSR-GUP black hole described by (31), with $\vartheta=\left(1-\frac{|P|}{\kappa}\right)^{2}$. Let $T$ denotes the black hole temperature, and the characteristic momentum being defined as $k_{B} T / c$ [20], then the mass becomes

$$
\tilde{M}=\frac{M}{\left(1-\left|\frac{k_{\beta} T}{c}\right| / \kappa\right)^{2}} \text {. }
$$


Fig. 2 Plot of the modified temperature-Hawking temperature relation for the $T_{H}^{D S R-G U P}$ with $\kappa=0.15,0.25$ and 0.35 where we set $\hbar=c=k_{B}=G=8 \pi=1$.

Fig. 3 Plot of the modified mass-temperature relation for the $M^{D S R-G U P}$ with $\kappa=0.15,0.25$ and 0.35 where we set

$\hbar=c=k_{B}=G=8 \pi=1$.
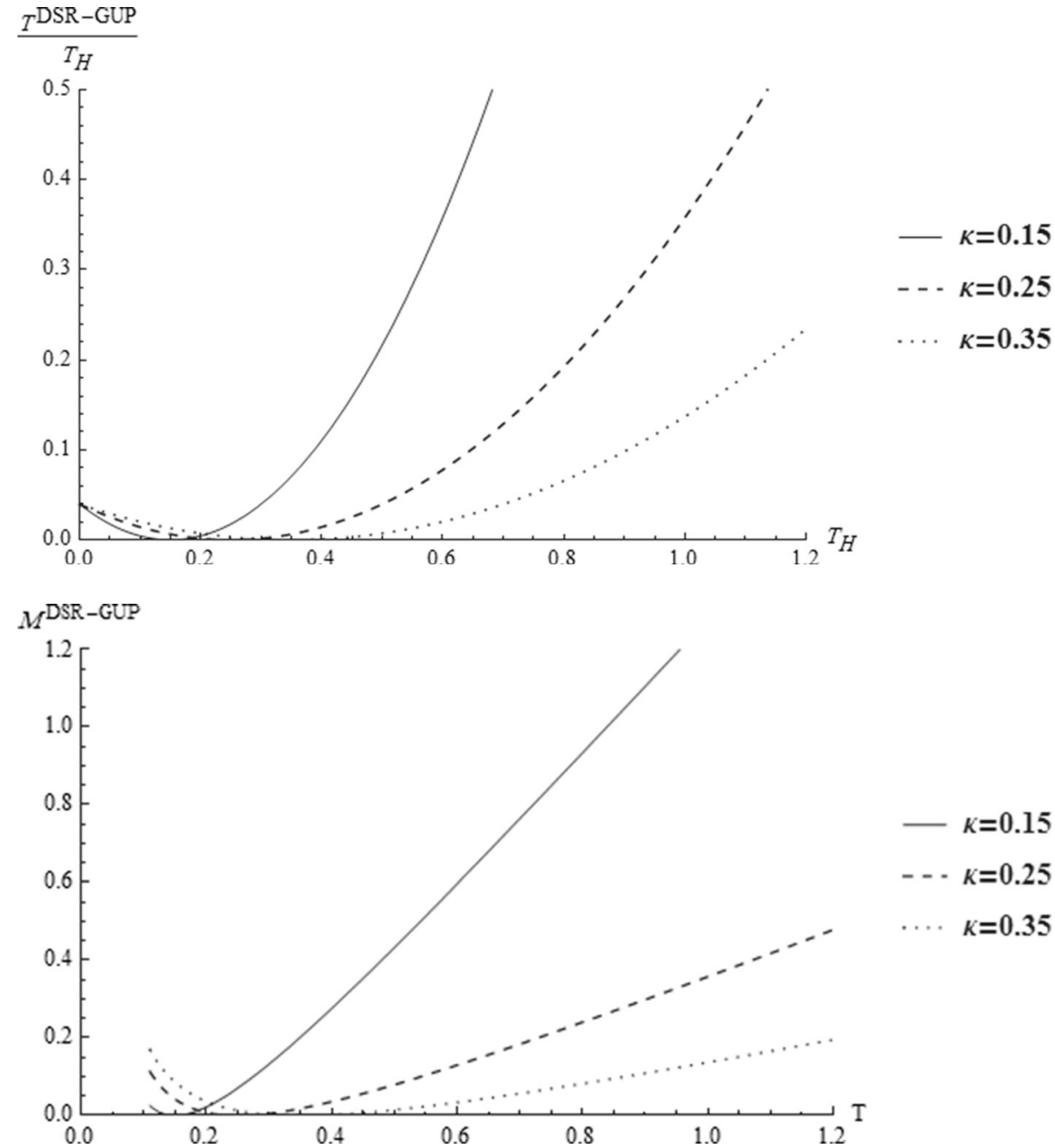

The horizon is determined by $r_{T}=2 G \tilde{M} / c^{2}$. Also, for modified temperature based on DSR-GUP from Hawking temperature becomes

$T_{H}^{D S R-G U P}=\frac{\hbar c^{3}}{8 \pi G k_{B} \widetilde{M}}=T_{H}\left(1+\frac{k_{\beta}^{2} T^{2}}{\kappa^{2} c^{2}}-\frac{2}{\kappa}\left|\frac{k_{\beta} T}{c}\right|\right)$.

In Fig. 2, we see that the modified Hawking temperature vs. temperature first tends to zero and next becomes increasing. Also, for decreasing $\kappa$, the minimum tends to zero.

As depicted in Fig. 2 that $T_{H}^{D S R-G U P}$ approaches to a minimum value at

$T \geq T_{0}=\frac{c \kappa}{k_{B}}$.

By solving Eq. (33) as a quadratic equation, we obtain the modified mass for DSR-GUP in the form

$M^{D S R-G U P}=\frac{\hbar c^{3}}{8 \pi G k_{B}}\left(\frac{1}{T}+\frac{k_{B}^{2} T}{\kappa^{2} c^{2}}-\frac{2 k_{B}}{\kappa c}\right)$.
Figure 3 shows that the modified mass vs. temperature is first decreasing and then increasing. For increasing $\kappa$, the minimum point shifts to higher temperatures.

Thus, the specific heat for the DSR-GUP black hole can be defined as

$$
\begin{aligned}
C^{D S R-G U P} & =c^{2} \frac{d M^{D S R-G U P}}{d T} \\
& =\frac{\hbar c^{5}}{8 \pi G k_{B}}\left(-\frac{1}{T^{2}}+\frac{k_{B}^{2}}{\kappa^{2} c^{2}}\right) .
\end{aligned}
$$

It is worth noting that the specific heat at temperature of Eq. (34) vanishes [30]. Figure 4 shows that the modified specific heat vs. temperature is increasing and tends to saturation point which is higher for smaller $\kappa$.

The entropy for the DSR-GUP black hole is finally obtained as

$$
\begin{aligned}
S^{D S R-G U P} & =\int \frac{C^{D S R-G U P}}{T} \mathrm{~d} T \\
& =\frac{\hbar c^{5}}{8 \pi G k_{B}}\left(\frac{1}{2 T^{2}}+\frac{k_{B}^{2}}{\kappa^{2} c^{2}} \operatorname{Ln}(T)\right) .
\end{aligned}
$$


Fig. 4 Plot of the modified specific heat-temperature relation for the $C^{D S R-G U P}$ with $\kappa=0.15,0.25$ and 0.35 where we set

$\hbar=c=k_{B}=G=8 \pi=1$.

Fig. 5 Plot of the modified entropy-temperature relation for the $S^{D S R-G U P}$ with

$\kappa=0.15,0.25$ and 0.35 where we set

$\hbar=c=k_{B}=G=8 \pi=1$.

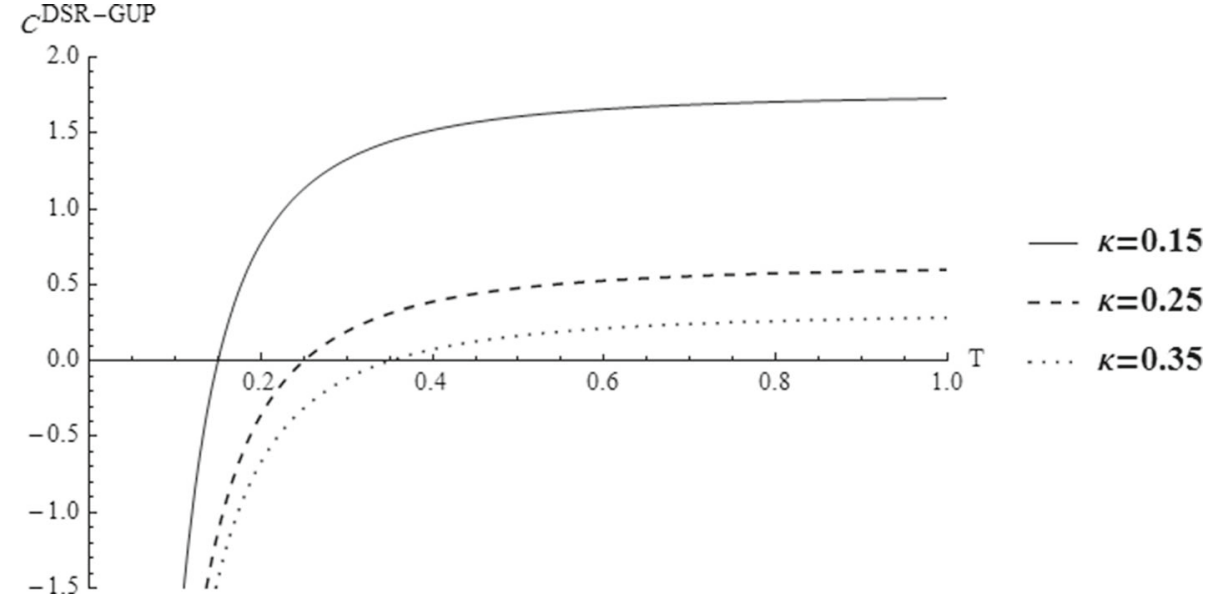

$S^{\mathrm{DSR}-\mathrm{GUP}}$

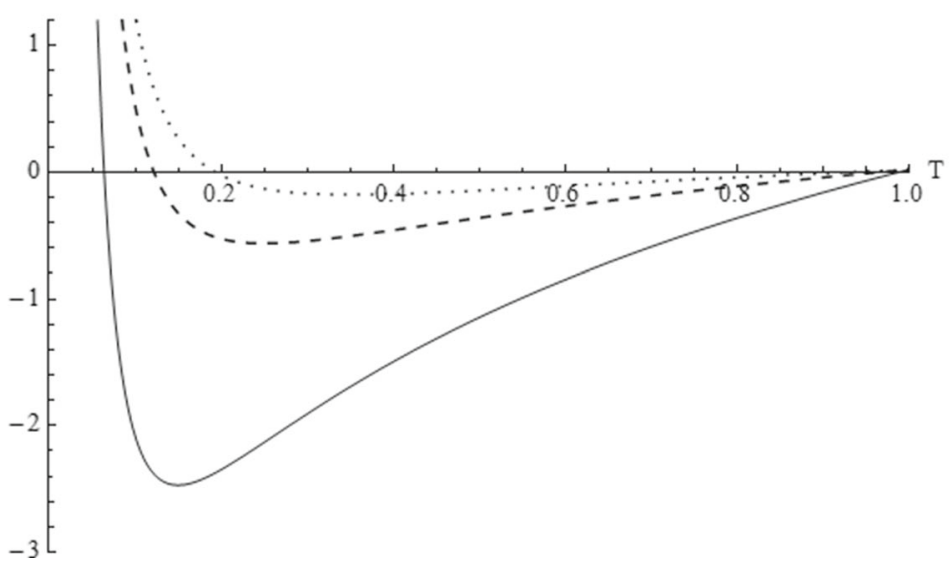

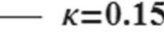

- - $K=0.25$

$\cdots \quad k=0.35$
Figure 5, modified entropy vs. temperature, represents a minimum and then tends to zero for large enough temperatures. In addition, Eqs. (33), (35) and (37) it is deduced that the minima of Hawking temperature, modified mass and mass and modified entropy all occur at $\mathrm{T}_{0}$ that it shows in Eq. (34).

$S\left(T_{0}\right)=\frac{\hbar k_{B} c^{3}}{16 \pi G \kappa^{2}}\left(1+2 \operatorname{Ln}\left(\frac{\kappa}{c} k_{B}\right)\right)$.

\section{Conclusions}

In this paper, we investigated the modified phase shift based on the COW experiment for DSR-GUP. Also, by studying the Einstein Bohr's photon box, we obtained modified Hawking temperature for DSR-GUP and some black hole properties including mass, specific heat, and entropy. We demonstrated that the COW experiment and Einstein Bohr's photon box due to the modified effective gravitational field and related to DSR-GUP. Notably, black hole properties depend on the temperature, that's why we have plotted them versus temperature. We have seen that the modified Hawking tempera- ture versus temperature first tends to zero and next becomes increasing. Also, for decreasing $\kappa$, the minimum tends to zero. Then, we have plotted the modified mass versus temperature which is first decreasing and then increasing. For increasing $\kappa$, the minimum point shifts to higher temperatures. After that, we have plotted the modified specific heat versus temperature which is increasing and tends to a saturation point which is higher for smaller $\kappa$. Finally, we have plotted the modified entropy versus temperature, which represents a minimum, and next tends to zero for large enough temperatures. In addition, it is seen Eqs. (33), (35), and (37) that the minima of modified Hawking temperature, modified mass, and modified entropy all occur at characteristic temperature $T_{0}$.

Acknowledgements The authors thank the referee for a thorough reading of our manuscript and for constructive suggestion. Hassan Hassanabadi and Jan Kříž are grateful for the institutional support of the Faculty of Science, University of Hradec Králové, research team "Mathematical physics and differential geometry"

Data Availability Statement This manuscript has associated data in a data repository. [Authors' comment: There is not any numerical calculation in this work.] 
Open Access This article is licensed under a Creative Commons Attribution 4.0 International License, which permits use, sharing, adaptation, distribution and reproduction in any medium or format, as long as you give appropriate credit to the original author(s) and the source, provide a link to the Creative Commons licence, and indicate if changes were made. The images or other third party material in this article are included in the article's Creative Commons licence, unless indicated otherwise in a credit line to the material. If material is not included in the article's Creative Commons licence and your intended use is not permitted by statutory regulation or exceeds the permitted use, you will need to obtain permission directly from the copyright holder. To view a copy of this licence, visit http://creativecomm ons.org/licenses/by/4.0/.

Funded by $\mathrm{SCOAP}^{3}$.

\section{References}

1. W. Heisenberg, F. Zeits. Phys. 43172 (1927)

2. M. Maggiore, Phys. Rev. D 49(10), 5182 (1994)

3. K. Konishi, G. Pauti, P. Provero, Phys. Lett. B 234, 276 (1990)

4. F. Scardigli, Phys. Lett. B 452(1-2), 39-44 (1999)

5. M. Maggiore, Phys. Lett. B 304, 63 (1993)

6. B.G. Sidharth, Found. Phys. Lett. 15(6), 577-583 (2002)

7. N. Farahani, H. Hassanabadi, W.S. Chung, L. Naderi, Commun. Theor. Phys. 72, 035404 (2020)

8. W.S. Chung, H. Hassanabadi, Phys. Lett. B 785, 127-131 (2018)

9. G. Amelino-Camelia, Int. J. Mod. Phys. D 11(10), 1643-1669 (2002)

10. J.L. Cortes, J. Gamboa, Phys. Rev. D 71(6), 065015 (2005)

11. A.W. Overhauser, R. Colella, Phys. Rev. Lett. 33, 1237-1239 (1974)

12. R. Colella, A.W. Overhauser, S.A. Werner, Phys. Rev. Lett. 34, 1472-1274 (1975)
13. A. Camacha, A. Camacho-Guardia, In AIP Conference Proceedings, vol. 1122, no. 1, pp. 209-212. AIP, (2009)

14. S. Hawking, Vol. 8. World Scientific Publishing Company, (1993)

15. Y. Aharonov, D. Rohrlich, Quantum Paradoxes (Wiley-Vch, Weinheim, 2005)

16. N. Bohr, Niels Bohr Collected Works, vol. 7 (Elsevier, Amsterdam, 1996), pp. 339-381

17. L.J. Garay, Int. J. Mod. Phys. A 10 (1995) 145-166, and the references therein

18. J. Kowalski-Glikman, S. Nowak, Class. Quantum Gravity 20(22), 4799 (2003)

19. W.S. Chung, H. Hassanabadi, M.S. Kang, H.H. Sung, J. Maeng, S. Lee, Y.I. Moon, Mod. Phys. Lett. A 34(07), 1950052 (2019)

20. L. Xiang, Y. Ling, Y.G. Shen, C.Z. Liu, H.S. He, L.F. Xu, Ann. Phys. 396, 334-350 (2018)

21. W.S. Chung, H. Hassanabadi, Prog. Theor. Exp. Phys. 12, $123 E 01$ (2019)

22. E. Maghsoodi, H. Hassanabadi, W.S. Chung, Prog. Theor. Exp. Phys. 8, 083E03 (2019)

23. A. Kempf, G. Mangano, R.B. Mann, Phys. Rev. D 52, 1108-1118 (1995)

24. D.V. Ahluwalia, Phys. Lett. A 275, 31-35 (2000)

25. K. Varjú, L.H. Ryder, Am. J. Phys. 68(5), 404-409 (2000)

26. S.A. Werner, H. Kaiser, M. Arif, R. Clothier, Phys. B 151(1-2), 22-35 (1988)

27. A. Saha, Phys. Rev. D 89(2), 025010 (2014)

28. A.C. de la Torre, A. Daleo, I. Garcia-Mata, Eur. J. Phys. 23(4), L15 (2002)

29. W. G. Unruh, G. I. Opat, No. UM-P-78/65. Melbourne Univ., (1979)

30. W.S. Chung, H. Hassanabadi, Phys. Lett. B 793, 451-456 (2019) 